\title{
THE ROLE OF RENAL BIOPSY AND IF IN CLASSIFYING NEPHRITIC SYNDROME
}

\author{
Anseena Kantirathingal Muhammed ${ }^{1}$, Lillykutty Pothen², Jayakumar Kavanakkattu Parameswaran³, Sankar $S^{4}$ \\ 1 Resident, Department of Pathology, Government Medical College, Kottayam. \\ ${ }^{2}$ Additional Professor, Department of Pathology, Government Medical College, Kottayam. \\ 3 Professor and HOD, Department of Nephrology, Government Medical College, Kottayam. \\ 4 Professor and HOD, Department of Pathology, Government Medical College, Kottayam.
}

\begin{abstract}
BACKGROUND
The primary role of the renal biopsy is to provide a diagnosis that allows the clinician to make an informed prognosis and assign lesion-specific therapy. Renal biopsy has become an indispensable tool in the investigation of medical diseases of the kidney because early diagnosis and treatment can prevent longterm complications. The use of Light microscopy and Immunofluorescence alone may be sufficient to diagnose common glomerular diseases encountered in clinical practice. Hence, the present study was conducted to study and evaluate the glomerular pathology on the basis of light microscopic and immunofluorescence findings in renal biopsies of patients presenting with features of nephritic syndrome and establish a diagnosis.
\end{abstract}

ABSTRACT

\section{MATERIALS AND METHODS}

A descriptive study was done to analyse the histopathology and immunofluorescence pattern of renal biopsies of patients presenting with features of Acute Glomerulonephritis. For this all renal biopsies received in the Department of Pathology from April 2015 to April 2016 which met the defined criteria were analysed.

\section{RESULTS}

Among the 31 cases studied, 9 (29\%) cases of Glomerulonephritis were Non-IgA mesangioproliferative Glomerulonephritis, 6 (20\%) cases were IgA Nephropathy, 9 (29\%) cases were Diffuse Endocapillary Proliferative Glomerulonephritis (DEPGN), 1 (3\%) case was Membranoproliferative Glomerulonephritis (MPGN), 2 (6\%) cases were of Lupus Nephritis and 4 (13\%) cases were Tubulointerstitial Nephritis (TIN). 25 (80\%) cases showed immunoglobulin deposits on immunofluorescence study. Of these, 10 $(40 \%)$ cases showed mesangial immunoglobulin deposits, 12 (48\%) cases showed granular immunoglobulin deposits along the capillary wall and $3(12 \%)$ cases showed linear immunoglobulin deposits along the capillary wall.

\section{CONCLUSION}

Immunofluorescence plays a vital role in elucidating the presumed pathogenesis in glomerular lesions and helps to arrive at a final diagnosis when combined with light microscopic findings.

\section{KEYWORDS}

Acute Glomerulonephritis; Light Microscopy; Immunoglobulin Deposits; Non-IgA Mesangioproliferative GN; IgA Nephropathy; MPGN; DEPGN; Lupus Nephritis.

HOW TO CITE THIS ARTICLE: Muhammed AK, Pothen L, Parameswaran JK, et al. The role of renal biopsy and IF in classifying nephritic syndrome. J. Evolution Med. Dent. Sci. 2017;6(93):6672-6679, DOI: 10.14260/jemds/2017/1446

\section{BACKGROUND}

Glomerular diseases constitute an important cause of morbidity and mortality and impose considerable burden on the already strained health services in developing countries. Glomerulonephritis is one of the few human disorders, in which animal studies have successfully unfolded its intricate nature. The diagnosis and classification of Glomerular diseases poses a great challenge to the pathologist owing to its complexity and variety of lesions. The advent of renal biopsy has helped in simplifying the diagnosis of Glomerular diseases. However, the use of light microscopy alone may not be sufficient to establish a final diagnosis. The application of Electron microscopy in the fifties and Immunofluorescence in the sixties have greatly clarified the pathogenesis and scientific basis of classification of glomerular disease.

'Financial or Other Competing Interest': None.

Submission 11-10-2017, Peer Review 21-11-2017,

Acceptance 27-11-2017, Published 11-12-2017.

Corresponding Author:

Dr. Lillykutty Pothen,

Additional Professor, Department of Pathology,

Government Medical College, Kottayam.

E-mail:glamskan@gmail.com

DOI: $10.14260 /$ jemds $/ 2017 / 1446$
In the present study, the Pattern of glomerular diseases presenting with haematuria, acute onset of oedema, red cell cast in urine, oliguria and mild-to-moderate hypertension (nephritic syndrome features) were analysed. The histological diagnoses enable the Nephrologists to separate the patients in such heterologous clinical settings into therapeutically and prognostically meaningful categories.

Hence, the present study was being conducted to study glomerular pathology on the basis of light microscopic (LM) and Immunofluorescence findings and to establish a diagnosis in cases presenting with nephritic syndrome.

\section{MATERIALS AND METHODS \\ Type of Study}

Descriptive study done to analyse the histopathology and immunofluorescence pattern of renal biopsies of patients presenting with features of acute glomerulonephritis.

\section{Study Setting}

Department of Pathology, Government Medical College, Kottayam during April 2015 to April 2016. 


\section{Sample Size}

All renal biopsies, which were presented with features of nephritic syndrome received in Department are analysed (Since the number of sample size is less as compared with the previous year statistics).

\section{Inclusion Criteria}

All cases of nephritic syndrome.

\section{Exclusion Criteria}

None.

\section{Study Procedure}

The study was approved by the Ethics Committee and the Research Board of our institution. Consent was taken from patients to obtain clinical details from each patient. All Patients with clinical features of nephritic syndrome were included in the study, which included 31 cases. The following information at the time of the renal biopsy was recorded, including: age of the patient, sex, haematuria, oliguria, renal failure, presence or absence of hypertension (defined as blood pressure $>140 / 90 \mathrm{mmHg}$ or the use of antihypertensive agents), 24-hour urine protein excretion, urine RBC, Hb. Total Count, Platelet, Serum Creatinine, Serum C3 levels, Serum ANA and dsDNA levels, ANCA and Anti-GBM antibodies.

Two cores of renal tissue were obtained by percutaneous renal biopsy. Of the two cores, one core was sent in normal saline for immunofluorescence and other core in 10\% neutral buffered formalin for light microscopy. The renal tissue sent in saline is frozen and is cut using ASP $300 \mathrm{~S}$ model cryostat and stained for immunoglobulin A, M, G, C3, C1q.

\section{Light Microscopy}

The renal tissue obtained in $10 \%$ neutral buffered formalin is kept for fixation for 8-12 hours and then processed in a Leica CM $1510 \mathrm{~S}$ model Histokinete and embedded in paraffin wax. Sections of 3-5 micron thickness was cut using Leica RM 2125 model rotatory microtome and stained, The routine stains used were Haematoxylin and Eosin, Periodic acid Schiff, Jones' reticulin and Masson's trichrome.

After studying the morphology and Immunofluorescence patterns were correlated with clinical and laboratory parameters, diagnosis was established. Glomerular lesions were classified into primary and secondary accordingly. Baseline demographical, clinical laboratory and histological data were recorded and were statistically analysed using the SPSS version 16.0 software (Chicago, IL, USA). Results were expressed as frequencies and percentage.

\section{RESULTS}

- Number of glomerulus in the biopsy cores ranged from 5 to 22 with a mean of $12 \pm 4.7$.

- $20(65 \%)$ were male and 11 (35\%) were female patients.

- $\quad$ The mean age was $38.8 \pm 14$ years, with a minimum age of 17 years and maximum age of 60 years.

- Maximum number of cases fell in the age group between 40-50 years.

- $71 \%$ cases presented with abrupt onset of macroscopic haematuria and $10 \%$ presented with acute onset of oedema and $19 \%$ presented with both haematuria and oedema.
- $\quad$ All the cases had microscopic haematuria with 3 cases showing RBC casts also.

- Elevated serum creatinine was present in $90 \%$ of the cases with a mean of $4.8 \pm 3.4$.

- Serum C3 levels was low in 35\% cases.

- 17 (55\%) cases of GN showed Diffuse Mesangioproliferative changes in the glomeruli. 9 (29\%) cases had Diffuse Endocapillary Proliferative changes. Membranoproliferative change was noted in 1 (3\%) case. Sclerosed Glomerulus was the glomerular change in $4(13 \%)$.

- $\quad 25(80 \%)$ cases of GN showed immunoglobulin deposits on immunofluorescence study, $6(20 \%)$ cases showed absent immunoglobulin deposits.

- $10(40 \%)$ cases showed mesangial immunoglobulin deposits, 12 (48\%) cases showed granular and 3 (12\%), cases showed linear immunoglobulin deposits along the capillary wall.

- The most frequent type of single immune deposits in various combination was C3 $(88 \%)$ followed by IgG (36\%).

- $\quad$ The most frequent combination of deposits were $\mathrm{IgG}+\mathrm{C} 3$ found in $12 \%$ cases.

- $25(81 \%)$ cases of GN were Primary Glomerular lesions, $2(6 \%)$ cases were Secondary Glomerular lesions and 4 (13\%) cases turned o

- ut to be acute-on-chronic Tubulointerstitial Nephritis (TIN).

- Lupus Nephritis constituted $100 \%$ of the secondary glomerular lesions (2 cases) and was serologically confirmed and showed Full House IF.

- $9(29 \%)$ cases of GN were Non IgA Mesangioproliferative Glomerulonephritis, 6 (20\%) cases were IgA Nephropathy, $9(29 \%)$ cases were DEPGN, 1 (3\%) case was MPGN, 2 (6\%) cases of Lupus Nephritis and 4 (13\%) cases were Acute on Chronic Tubulointerstitial Nephritis (TIN).

- 2 cases of Lupus Nephritis presented as Diffuse Mesangioproliferative Lesions and 1 case of IgA Nephropathy presented as crescentic glomerular lesion.

\section{DISCUSSION}

The present knowledge of the pathology of renal diseases has been derived to a large extent after the introduction of percutaneous needle biopsy of the kidney and the systematic study of these small samples of renal tissue by light microscopy, electron microscopy and immunofluorescence microscopy. The primary role of the renal biopsy is to provide a diagnosis that allows the clinician to make an informed prognosis and assign lesion-specific therapy. Renal biopsy has become an indispensable tool in the investigation of medical diseases of the kidney because early diagnosis and treatment can prevent long-term complications. In the present study, the histopathological features \& immunofluorescence pattern of renal biopsies in patients who presented with features of Acute Glomerulonephritis were analysed and diagnosis was established. The study included 31 renal biopsies from patients who presented with features of nephritic syndrome. Histopathological \& immunofluorescence findings were correlated with clinical and laboratory data. 


\begin{tabular}{|c|c|c|}
\hline Study & Year & Mean Age \\
\hline Kim BS et al $^{1}$ & $2009(\mathrm{n}-156)$ & $38.3 \pm 11.9$ \\
\hline Hossain T et al $^{2}$ & $2011(\mathrm{n}-78)$ & 30.67 years \\
\hline Borphukan S et $\mathrm{al}^{3}$ & $2016(\mathrm{n}-48)$ & $37 \pm 15.7$ years \\
\hline Present study & $2016(\mathrm{n}-31)$ & $38.8 \pm 14$ years \\
\hline \multicolumn{2}{|c|}{ Table 1. Comparison of Mean Age of Patients at } \\
Presentation
\end{tabular}

In the present study, the mean age of patients who presented with Acute Glomerulonephritis was $38.8 \pm 14$ years, which is comparable with the studies of Kim B S et al ${ }^{1}$ and Borphukan $\mathrm{S}$ et al. ${ }^{3}$ In the study by Hossain $\mathrm{T}$ et $\mathrm{al},{ }^{2}$ mean age was 30.67 years, which was slightly in the lower range.

\begin{tabular}{|c|c|c|c|}
\hline Study & Year & Male (\%) & Female (\%) \\
\hline $\begin{array}{c}\text { Rahul Mannan } \\
\text { et al }{ }^{4}\end{array}$ & $2012(n-52)$ & 62 & 38 \\
\hline Buch AC et al ${ }^{5}$ & $2015(n-32)$ & 57.3 & 42.6 \\
\hline $\begin{array}{c}\text { Shazia Hamid } \\
\text { et al } 6\end{array}$ & $2015(n-65)$ & 62 & 38 \\
\hline Present study & 2016 & 65 & 35 \\
\hline
\end{tabular}

In the present study, $65 \%$ of cases occurred in males and $35 \%$ in the females. This is similar to other similar studies.

But in Lupus Nephritis females predominated over males. In the present study, females constituted $100 \%$ of Lupus Nephritis and this was correlating with the studies by Golay $\mathrm{V}$ et $\mathrm{al}^{7}(82 \%)$, Jeganathan $\mathrm{J}$ et $\mathrm{al}^{8}(87 \%)$ and Zhou $\mathrm{F}$ D et $\mathrm{al}^{9}$ (84\%).

\section{Presenting Complaints}

$71 \%$ patients presented with macroscopic haematuria, 10\% patients had acute onset of oedema whereas $19 \%$ presented with both haematuria and acute onset of oedema, which is not in league with studies by Natarajan $\mathrm{G}$ et $\mathrm{al}^{10}$ and Shazia Hamid et $\mathrm{al}^{6}{ }^{6}$ in which acute onset of oedema was the predominant presentation. This difference may be due to the lesser sample size and the population bias in the present study.

In patients those who also had associated renal failure, the most common aetiology of GN were Non-IgA Mesangioproliferative GN followed by Crescentic GN and Chronic Sclerosing GN.

$67.7 \%$ patients had hypertension, which is comparable with studies of Shazia Hamid et al (81.53\%) and Natarajan G et al $(74 \%)$.

$7 \%$ cases had history of Chronic Kidney Disease, and all the cases turned out to be Non-IgA Mesangioproliferative
GN and this may be due to the fact that Non-IgA Mesangioproliferative GN might have caused the chronic Kidney Disease.

1 case presented with history of Chronic Viral Infection (Hepatitis C) and it was histologically MPGN.

$2(6 \%)$ cases presented with past history of acute infection (both were cases of acute respiratory tract infection) and histologically 1 case was DEPGN and other case was Non-IgA Mesangioproliferative GN.

\section{Laboratory Parameters}

Hb values ranged from 7.1 to $14 \mathrm{~g} / \mathrm{dL}$ with a mean of $10.7 \pm$ 1.78 and $81 \%$ patients had anaemia (normocytic normochromic anaemia in all the cases.) Total Count was elevated in 6 cases, with $4(66.6 \%)$ cases proved to be as Crescentic GN.

\section{Urine Microscopy}

All the cases had microscopic RBCs with 3 showing RBC casts also.

\section{4-Hour Proteinuria}

$38.7 \%$ cases presented with proteinuria more than $3 \mathrm{~g} / \mathrm{dL}$ and $61.3 \%$ cases presented with proteinuria less than $3 \mathrm{~g} / \mathrm{dL}$. This is lower than in studies by Shazia Hamid et $\mathrm{al}^{6}(75.4 \%$ \& $24.6 \% \%)$ and Natarajan G et al ${ }^{10}(60 \%$ \& $40 \%)$.

\section{Serum Creatinine Value}

Elevated serum creatinine was noted in $90 \%$ of the cases with a mean of. $4.8 \pm 3.4$. Of these, $67 \%$ cases showed serum creatinine $>3 \mathrm{mg} / \mathrm{dL}$, which was in concordance with study by Natarajan $\mathrm{G}$ et $\mathrm{al}^{10}(60 \%) .35 \%$ cases showed low serum C3 level, which was low as compared to the study by Natarajan G et al ${ }^{10}(66 \%)$.

\section{ANA, dsDNA Antibody}

ANA, dsDNA Antibodies were evaluated in 6 cases and it was positive in $3(50 \%)$ cases.

\section{Anti-GBM Assay}

Anti-GBM Assay was done in 3 cases and it was found to be negative.

\section{C-ANCA \& P-ANCA}

C-ANCA \& P-ANCA was assessed in 7 cases and it was negative in all the 7 cases.

\section{Histomorphology of Glomerular Lesions}

\begin{tabular}{|c|c|c|c|c|c|c|}
\hline Study & Year & $\begin{array}{c}\text { Diffuse } \\
\text { Mesangioproliferative } \\
\text { (\%) }\end{array}$ & $\begin{array}{c}\text { Crescentic } \\
\text { \% }\end{array}$ & $\begin{array}{c}\text { Membranoproliferative } \\
\text { (\%) }\end{array}$ & $\begin{array}{c}\text { Diffuse } \\
\text { Endocapillary } \\
\text { Proliferative\% }\end{array}$ & $\begin{array}{c}\text { Sclerosed } \\
\text { Glomerulus } \\
\text { (\%) }\end{array}$ \\
\hline $\begin{array}{c}\text { Balakrishnan N } \\
\text { et al }{ }^{11}\end{array}$ & $2003(\mathrm{n}-826)$ & 48 & 30 & 6 & 8 & 4 \\
\hline Present & 2016 & 41.9 & 19.4 & 3.2 & 19.4 & 12.9 \\
\hline \multicolumn{7}{|r}{ Table 3. Comparison of Histomorphology of Glomerular Lesions } \\
\hline
\end{tabular}

In the present study, Diffuse Mesangioproliferative Glomerular lesions constituted the highest number of cases (41.9\%). This was similar to the study done by Balakrishnan
$\mathrm{N}$ et al.11 1 (3.2) case showed both Diffuse Mesangioproliferative and Diffuse Endocapillary Proliferative changes in the glomeruli. 
Out of 6 cases of Crescentic glomerular lesions, 2 (33.3\%) cases showed Cellular+Fibrocellular+Fibrous Crescents. In 1 $(16.7 \%)$ case each, the crescents were Fibrocellular + Fibrous, Cellular, Fibrocellular Crescent + Fibrinoid Necrosis and Fibrocellular Crescent.

\section{Immunoglobulin Deposits}

$25(80 \%)$ cases of GN showed immunoglobulin deposits on immunofluorescence study, 6 (20\%) cases showed absent immunoglobulin deposits. Of the 25 Immunofluorescence positive cases, $10(40 \%)$ cases showed mesangial immunoglobulin deposits which is similar to the study by Hossain $\mathrm{T}$ et al, ${ }^{2} 12$ (48\%) cases showed granular immunoglobulin deposits along the capillary wall. 3 (12\%), cases showed linear immunoglobulin deposits along the capillary wall. The most frequent type of single immune deposits in various combination was C3 $(88 \%, \mathrm{n}=25)$ followed by IgG (36\%), which is similar to study by Hossain $\mathrm{T}$ et al. ${ }^{2}$ The most frequent combination of deposits were IgG $+\mathrm{C} 3$ found in 3 cases $(12 \%, n=25)$, which is similar to study by Hossain $\mathrm{T}$ et al. $^{2}$

\section{Aetiology of Diffuse Mesangioproliferative Glomerular Lesions \& Crescentic Glomerular Lesions}

Of the 13 cases of Diffuse Mesangioproliferative Glomerular Lesions, 4 (30.7\%) cases were IgA Nephropathy, 7 (53.8\%) cases were Non-IgA Mesangioproliferative Glomerulonephritis and $2(14.3 \%)$ cases turned out to be Class IV Lupus Nephritis. 5 (83\%) cases of 6 crescentic glomerular lesions were Pauci-immune Glomerulonephritis (negative for ANCA) and $1(17 \%)$ case turned out to be IgA Nephropathy on IF study. All 6 cases with Diffuse Endocapillary Proliferative change were DEPGN.

1 case with both Diffuse Endocapillary and Diffuse Mesangial Proliferative glomerular change was confirmed as IgA Nephropathy.

\section{Aetiology of Glomerular Lesions}

$25(81 \%)$ cases were Primary Glomerular lesions, 2 (6\%) cases were Secondary Glomerular lesions and 4 (13\%) cases turned out to be Chronic Sclerosing Glomerulonephritis.

\begin{tabular}{|c|c|c|}
\hline Study & Year & Primary Lesions (\%) \\
\hline Chan KW et al & $1999(n-46)$ & 56 \\
\hline M Shawarby et al & $2010(n-108)$ & 54 \\
\hline Harish Chandra et al & $2013(n-85)$ & 92 \\
\hline Present Study & 2016 & 81 \\
\hline \multicolumn{3}{|c|}{$\begin{array}{l}\text { Table 4. Comparison of Frequency of Primary } \\
\text { Glomerular Lesions }\end{array}$} \\
\hline
\end{tabular}

In the present study, primary glomerular lesions constituted $81 \%$ of the total cases which was in concordance with other studies. Among the Primary Glomerular lesions, 7 (28\%) cases were Non-IgA Mesangioproliferative Glomerulonephritis, 6 (24\%) cases were IgA Nephropathy, 6 (24\%) cases were Diffuse Endocapillary Proliferative Glomerulonephritis (DEPGN), 5 (20\%) cases were Pauciimmune Crescentic Glomerulonephritis and 1 (4\%) case was Membranoproliferative Glomerulonephritis (MPGN).

\begin{tabular}{|c|c|c|}
\hline Study & Year & $\begin{array}{c}\text { Secondary Lesions } \\
(\%)\end{array}$ \\
\hline Chan K W et al ${ }^{12}$ & $1999(n-46)$ & 34 \\
\hline M Shawarby et al ${ }^{13}$ & $2010(n-108)$ & 46 \\
\hline Harish Chandra et al 14 & $2013(\mathrm{n}-85)$ & 8 \\
\hline Present study & 2016 & 6 \\
\hline \multicolumn{3}{|c|}{$\begin{array}{l}\text { Table 5. Comparison of Frequency of Secondary } \\
\text { Glomerular Lesions }\end{array}$} \\
\hline
\end{tabular}

In the present study, secondary glomerular lesions constituted $6 \%$ of cases which is comparable with the study by Harish Chandra et al. ${ }^{14}$

In this study, Lupus Nephritis constituted $100 \%$ of the secondary glomerular lesions and was serologically confirmed and showed Full House IF, and is in concordance with other studies by Balakrishnan $\mathrm{N}$ et al11 Golay $\mathrm{V}$ et al 7and Chan K W et al, ${ }^{2}$ where lupus nephritis constituted $100 \%$ or near to it.

In the present study, the mean age of patients who presented with Acute Glomerulonephritis was $38.8 \pm 14$ years, which is comparable with the studies of Kim B S et ${ }^{1}$ and Borphukan $\mathrm{S}$ et al. ${ }^{3}$ In the study by Hossain $\mathrm{T}$ et $\mathrm{al},{ }^{2}$ mean age was 30.67 years, which was slightly in the lower range.

In the present study, $65 \%$ of cases occurred in males and $35 \%$ in the females. This is similar to other studies. But in Lupus Nephritis females predominated over males. In the present study, females constituted $100 \%$ of Lupus Nephritis and this was correlating with the studies by Golay $\mathrm{V}$ et $\mathrm{al}^{7}$ (82\%), Jeganathan J et $\mathrm{al}^{8}(87 \%)$ and Zhou F D et $\mathrm{al}^{9}(84 \%)$.

$71 \%$ patients presented with macroscopic haematuria, $10 \%$ patients had acute onset of oedema whereas 19\% presented with both haematuria and acute onset of oedema, which is not in league with studies by Natarajan G et al ${ }^{10}$ and Shazia Hamid et al,6 where acute onset of oedema was the predominant clinical presentation. This difference may be due to the lesser sample size and the population bias in the present study.

In patients those who had associated renal failure, the most common aetiology of GN was DEPGN followed by Tubulointerstitial Nephritis (TIN).

Hypertension was present in majority of GN which is comparable with studies of Shazia Hamid et al ${ }^{6}(81.53 \%)$ and Natarajan G et al 10 (74\%).

$7 \%$ cases had history of Chronic Kidney Disease, and all the cases turned out to be Non-IgA Mesangioproliferative GN and this may be due to the fact that Non-IgA Mesangioproliferative GN might have caused the chronic Kidney Disease.

1 case presented with history of Chronic Viral Infection (Hepatitis C) and it was histologically MPGN. 2 (6\%) cases presented with past history of acute infection (both were cases of acute respiratory tract infection) and histologically 1 case was DEPGN and other case was Non-IgA Mesangioproliferative GN.

$\mathrm{Hb}$ values ranged from 7.1 to $14 \mathrm{~g} / \mathrm{dL}$ with a mean of 10.7 \pm 1.78 and $81 \%$ patients had anaemia (normocytic normochromic anaemia in all the cases.) Total WBC Count was elevated in 6 cases.

All the cases had microscopic haematuria with 3 cases showing RBC casts also. $38.7 \%$ cases presented with proteinuria more than $3 \mathrm{~g} / \mathrm{dL}$ and $61.3 \%$ cases presented with proteinuria less than $3 \mathrm{~g} / \mathrm{dL}$, this is lower than the 
studies by Shazia Hamid et $\mathrm{al}^{6}(75.4 \%$ \& $24.6 \%)$ and Natarajan G et al ${ }^{10}(60 \%$ \& 40\%).

Elevated serum creatinine was noted in $90 \%$ of the cases with a mean of $4.8 \pm 3.4$. Of these, $67 \%$ cases showed serum creatinine $>3 \mathrm{mg} / \mathrm{dL}$, which was in concordance with the study by Natarajan $\mathrm{G}$ et al (60\%).

$5 \%$ cases showed low serum C3 level, which was slightly lower compared to the study by Natarajan $G$ et al (66\%). DEPGN showed the maximum reduction in C3. ANA, dsDNA Antibodies were positive in $3(50 \%)$ cases. Anti-GBM Assay was negative in all the cases. C-ANCA \& P-ANCA was negative in all the cases.

In the present study, Diffuse Mesangioproliferative Glomerular lesions constituted the highest number of cases (55\%), this was similar to study done by Balakrishnan $\mathrm{N}$ et al. ${ }^{11}$ Crescents were noted in the glomeruli in 6 cases, In the 3 $(50 \%)$ cases, it was associated with Diffuse Mesangioproliferative Glomerular lesions and in the rest, it was associated with Diffuse Endocapillary Proliferative Glomerular Lesions.

$25(80 \%)$ cases of GN showed immunoglobulin deposits on immunofluorescence study, $6(20 \%)$ cases showed absent immunoglobulin deposits. Of the 25 Immunofluorescence positive cases, $10(40 \%)$ cases showed mesangial immunoglobulin deposits which is similar to study by Hossain $\mathrm{T}$ et $\mathrm{al}^{2} 12$ (48\%) cases showed granular immunoglobulin deposits along the capillary wall. 3 (12\%) cases showed linear immunoglobulin deposits along the capillary wall. The most frequent type of single immune deposits in various combination was C3 $(88 \%, \mathrm{n}=25)$ followed by IgG (36\%), which is similar to study by Hossain T et al. ${ }^{2}$ The most frequent combination of deposits were IgG+C3 found in 3 cases $(12 \%$, which is similar to study by Hossain $\mathrm{T}$ et al. ${ }^{2}$

\section{Aetiology of Diffuse Mesangioproliferative Glomerular Lesions}

Of the 17 cases of Diffuse Mesangioproliferative Glomerular Lesions, 6 (35\%) cases were IgA Nephropathy, 9 (53\%) cases were Non-IgA Mesangioproliferative Glomerulonephritis and 2 (12\%) cases turned out to be Class IV Lupus Nephritis. All 9 cases with Diffuse Endocapillary Proliferative change in the glomerulus turned out to be DEPGN.

\section{Aetiology of Glomerular Lesions}

25 (81\%) cases were Primary Glomerular lesions, 2 (6\%) cases were Secondary Glomerular lesions and $4(13 \%)$ cases turned out to be Tubulointerstitial Nephritis (TIN).

\begin{tabular}{|c|c|c|}
\hline Study & Year & Primary Lesions (\%) \\
\hline Chan KW et al & $1999(\mathrm{n}-46)$ & 56 \\
\hline M. Shawarby et al & $2010(\mathrm{n}-108)$ & 54 \\
\hline Harish Chandra et al & $2013(\mathrm{n}-85)$ & 92 \\
\hline Present Study & 2016 & 81 \\
\hline \multicolumn{3}{|c|}{ Table 6. Comparison of Frequency of Primary } \\
Glomerular Lesions \\
\hline
\end{tabular}

In the present study, primary glomerular lesions constituted $81 \%$ of the total cases which was in concordance with other studies. Among the Primary Glomerular lesions, 9 (36\%) cases were Non-IgA Mesangioproliferative Glomerulonephritis, 6 (24\%) cases were IgA Nephropathy, 9 $(36 \%)$ cases were Diffuse Endocapillary Proliferative
Glomerulonephritis, and 1 (4\%) case was Membranoproliferative Glomerulonephritis (MPGN).

In the present study, secondary glomerular lesions constituted $6 \%$ of cases which is only comparable with the study by Harish Chandra et al. In this study, secondary glomerular lesions were constituted by Lupus Nephritis and this was serologically confirmed and showed Full House IF pattern, and this is in concordance with other studies by Balakrishnan $\mathrm{N}$ et al, ${ }^{11}$ Golay $\mathrm{V}$ et $\mathrm{al}^{7}$ and Chan $\mathrm{K} \mathrm{W}$ et al,12 where lupus nephritis constituted the major proportion of secondary GN.

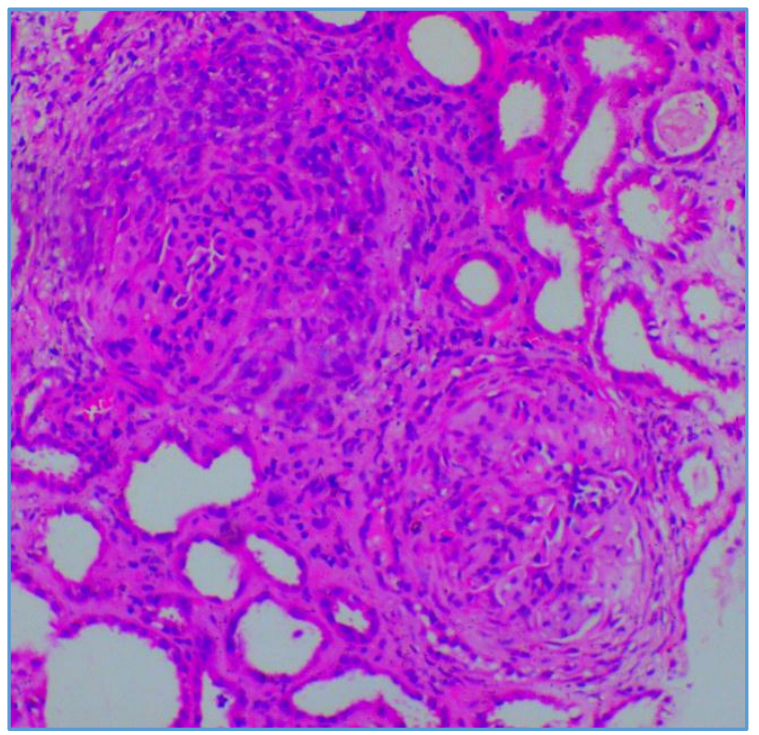

Figure 1. Photomicrograph Showing Cellular \& Fibrocellular Crescents (20 X) (H \& E Stain)

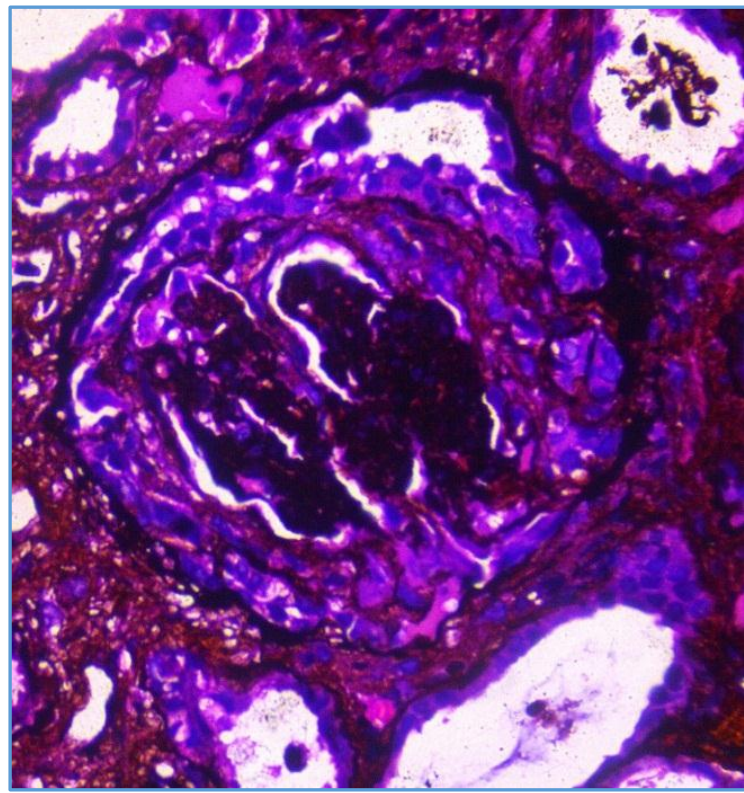

Figure 2. Photomicrograph Showing Cellular Crescent (40X) Jones' Reticulin Stain 


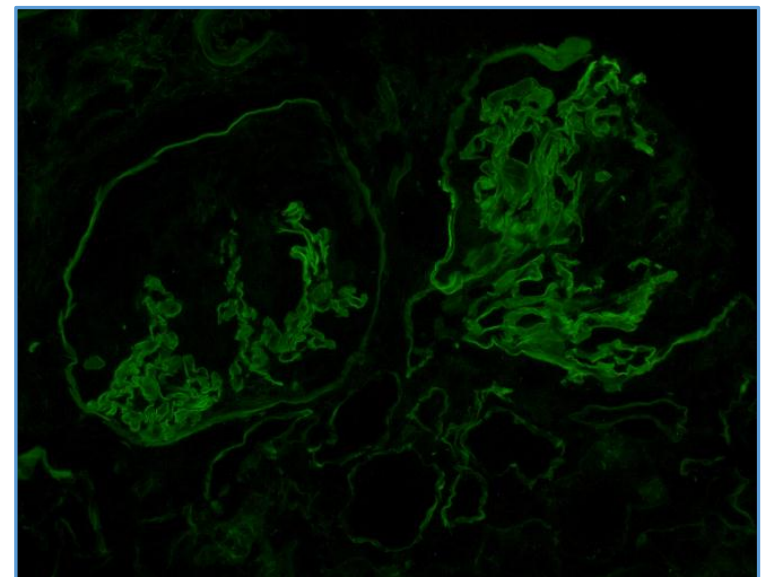

Figure 3. Photomicrograph Showing IgG 2+ Granular Deposits in Crescents (40 X) -IF

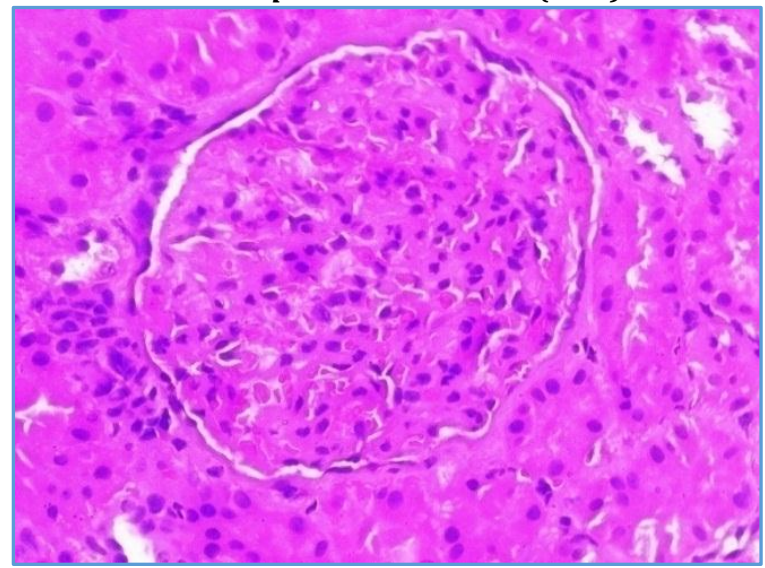

Figure 4. Photomicrograph Showing Diffuse Mesangioproliferative Glomeruli (40 X) in IgA Nephropathy (H\& E Stain)

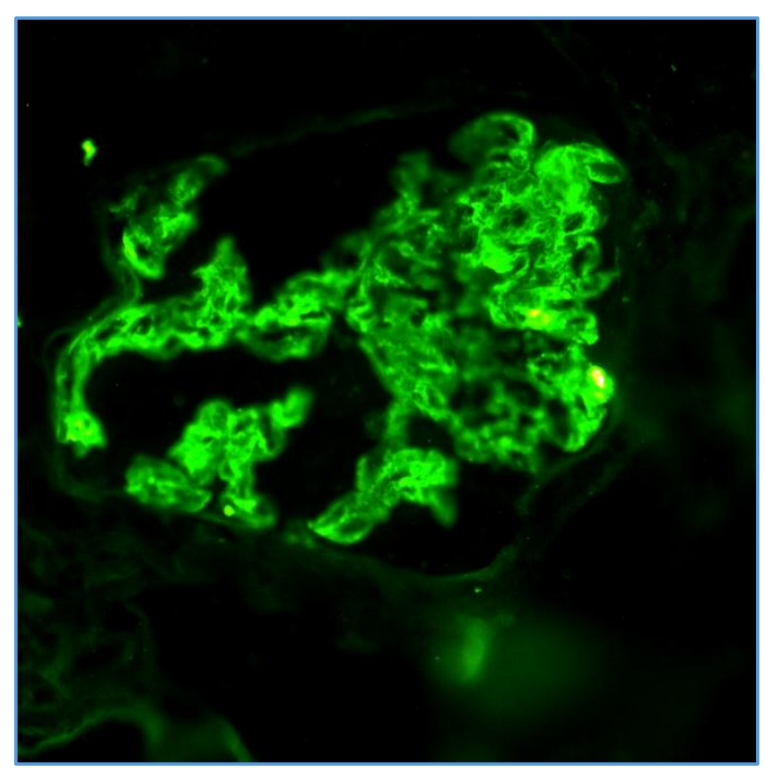

Figure 5. Photomicrograph Showing Granular IgA $3+$ Deposits (40 X) in IgA Nephropathy-IF

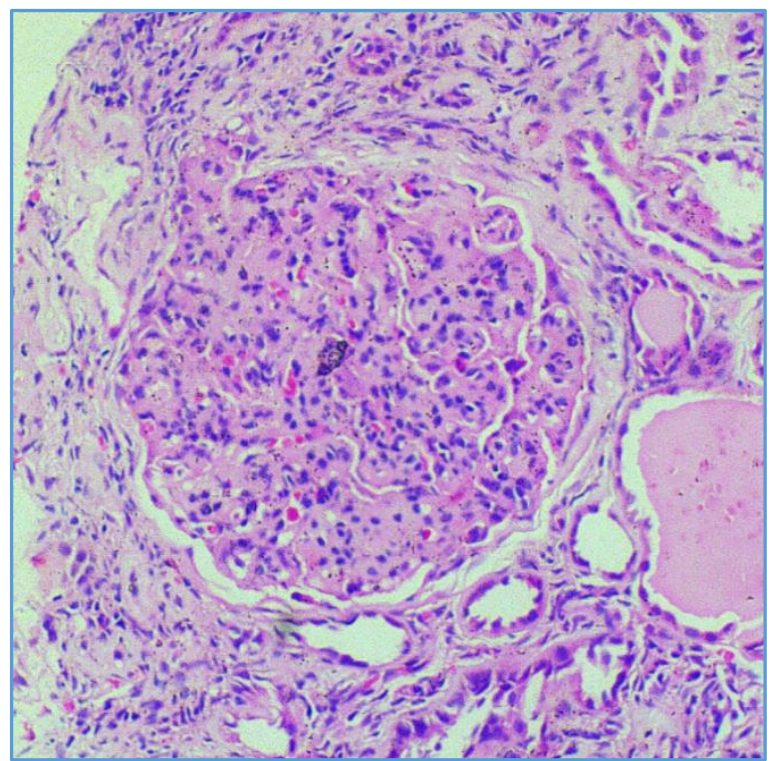

Figure 6. Photomicrograph Showing

Membranoproliferative Change in the Glomerulus (40 X) (H \&E Stain)

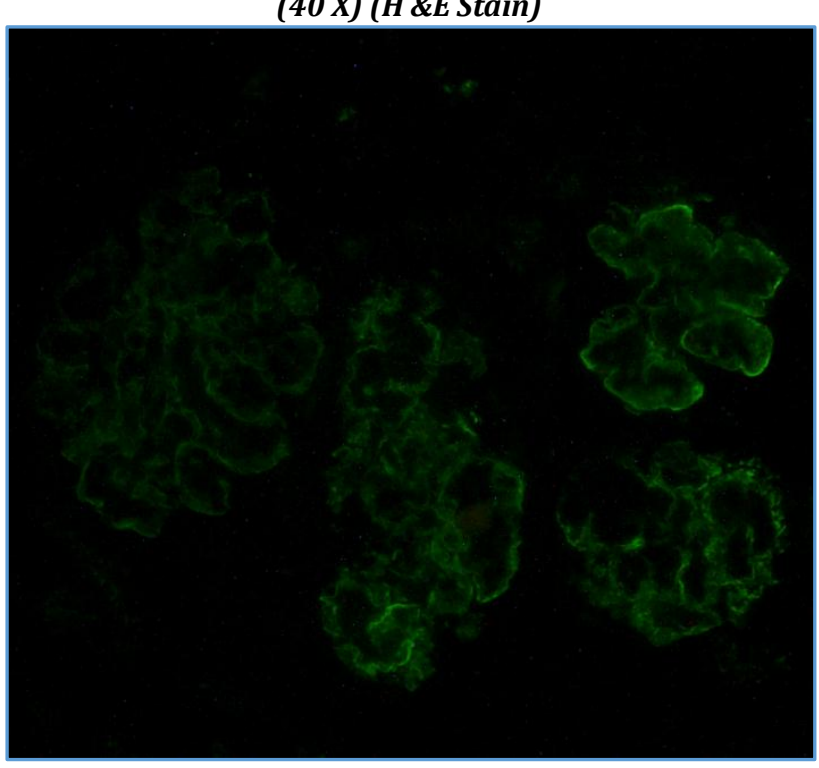

Figure 7. Photomicrograph Showing IgG 2+ Granular deposits Along the Capillary wall (20X) in MPGN-IF

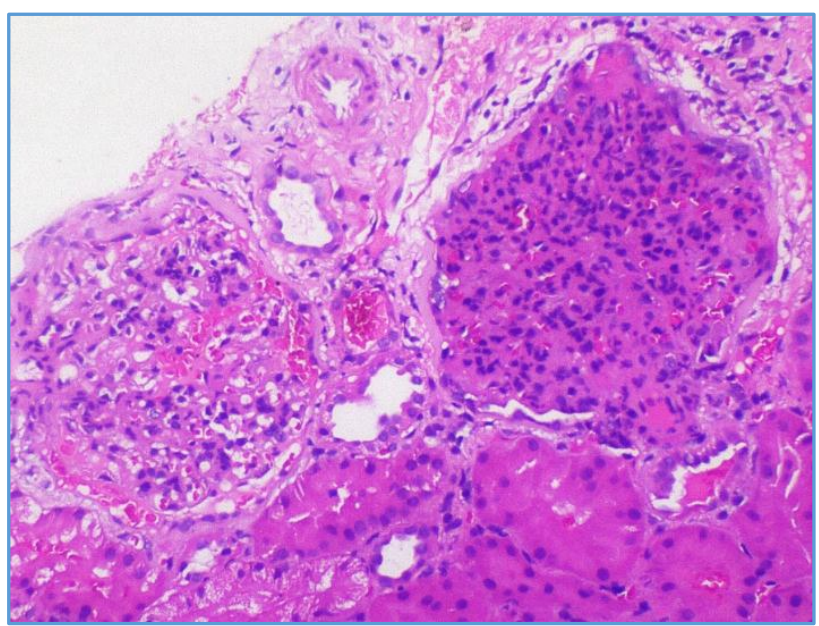

Figure 8. Photomicrograph Showing Glomerulus in DEPGN 20X (H \&E) 


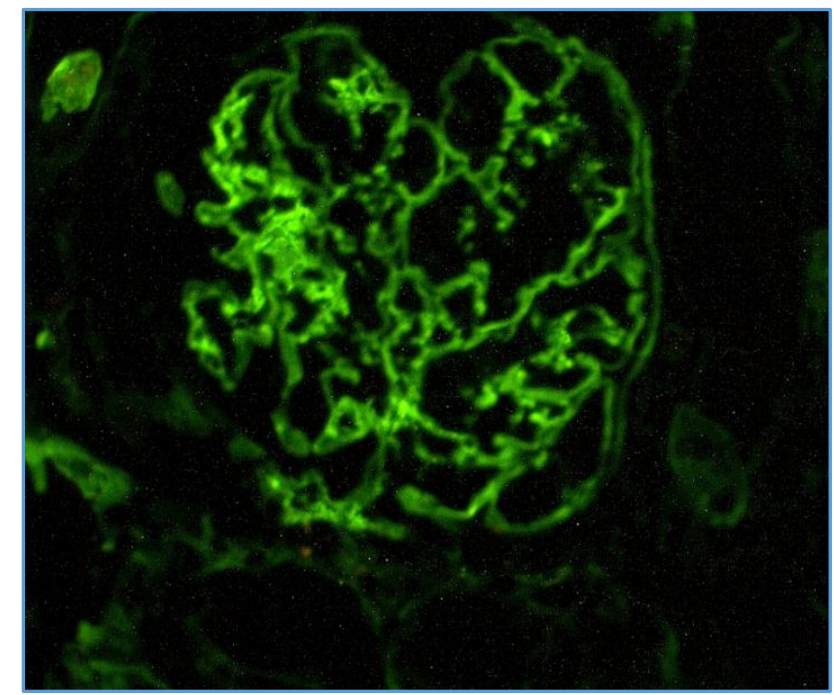

Figure 9. Photomicrograph Showing C3 2+ Linear Deposits Along the Capillary wall (4OX) in DEPGN-IF

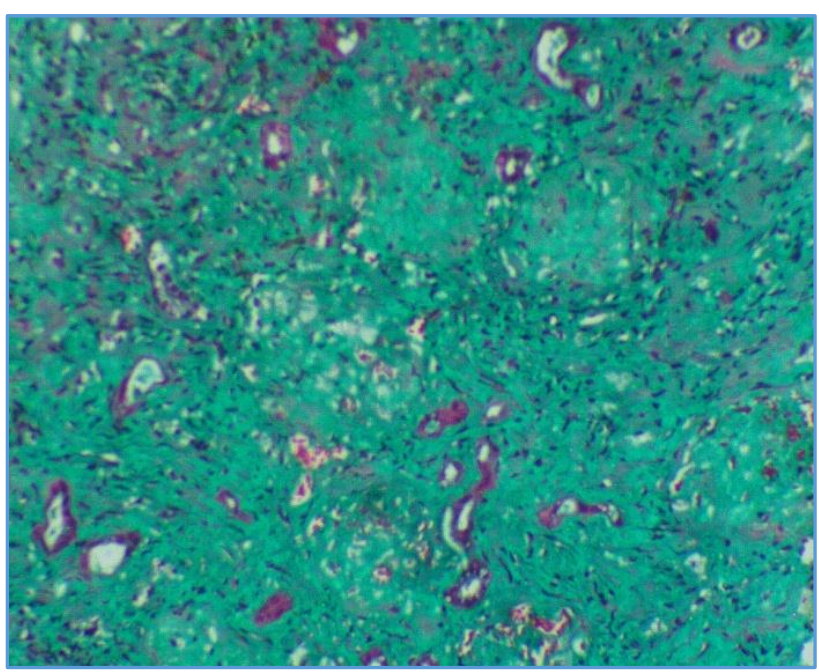

Figure 10. Interstitial Fibrosis As Shown By Masson's Trichrome (40 X)

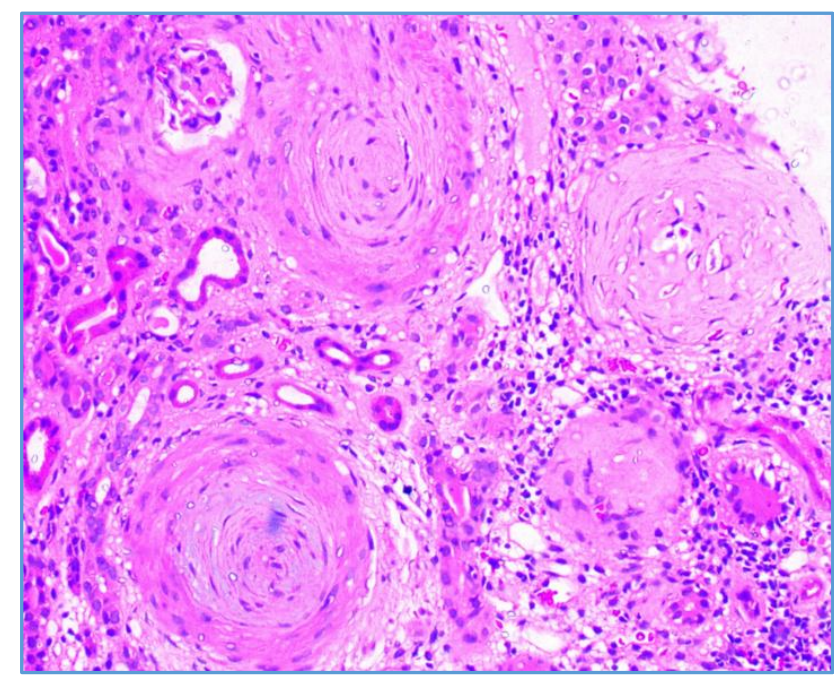

Figure 11. Hyperplastic Arteriolosclerosis \& Interstitial Inflammation 40 X (H\&E Stain)

\section{CONCLUSION}

Lesions were grouped into primary glomerular lesions and glomerular lesions secondary to systemic diseases. Diffuse Mesangioproliferative Glomerulonephritis was the commonest diagnosis in our study followed by DEPGN. NonIgA Mesangioproliferative Glomerulonephritis constituted the majority of GN followed by IgA nephropathy (as evidenced by IF study). Immunofluorescence findings were helpful in diagnosis of Lupus Nephritis and IgA Nephropathy.

Hence, we conclude that Immunofluorescence plays a vital role in elucidating the presumed pathogenesis in glomerular lesions and helps to arrive at a final diagnosis when combined with light microscopic findings.

\section{REFERENCES}

[1] Kim BS, Kim YK, Shin YS, et al. Natural history and renal pathology in patients with isolated microscopic hematuria. Korean J Intern Med 2009;24(4):356-61.

[2] Hossain MT, Begam M, Rahman AJE, et al. Immmune deposits in glomerular disease and their clinical, histopathological and immunopathological correlation. Bangladesh J Pathol 2011;26(1):14-9.

[3] Borphukan S, Gogoi S, Baruah D. Pattern of glomerular diseases in patients with significant proteinuria: a clinicopathological study from upper Assam. J Evid Based Med Healthc 2016;3(54):2756-61.

[4] Mannan R, Bhasin TS, Singh PA, et al. The pattern of glomerulonephritis in the north Indian gangetic plaina 13-year epidemiological study. Journal of Clinical and Diagnostic Research 2012;6(5):855-8.

[5] Buch AC, Sood SK, Bamanikar SA, et al. Role of direct immunofluorescence in the diagnosis of glomerulonephritis. Med J DY Patil Univ 2015;8(4):452-7.

[6] Hamid S, Kumar V, Badgal A, et al. Clinical profile of acute glomerulonephritis in north India: a single centre study. Journal of Evolution of Medical and Dental Sciences 2015;4(43):7458-60.

[7] Golay V, Trivedi M, Abraham A, et al. The spectrum of glomerular diseases in a single center: a clinicopathological correlation. Indian J Nephrol 2013;23(3):168-75.

[8] Jeganathan J, Kumar S, Khalid $M$, et al. Pattern of glomerular diseases in a tertiary care center in south India: a prospective study. Saudi J Kidney Dis Transpl 2013;24(1):168-71.

[9] Zhou FD, Shen HY, Chen M, et al. The renal histopathological spectrum of patients with nephrotic syndrome: an analysis of 1523 patients in a single Chinese centre. Nephrol Dial Transplant 2011;26(12):3993-7.

[10] Natarajan G, Ramanathan S, Jeyachandran D, et al. Follow-up study of post-infectious glomerulonephritis in adults: analysis of predictors of poor outcome. Saudi J Kidney Dis Transpl 2014;25(6):1210-6.

[11] Balakrishnan N, John GT, Korula A. Spectrum of biopsy proven renal disease and changing trends at a tropical tertiary care centre 1990-2001. Indian J Nephrol 2003;13(1):29-35. 
[12] Chan KW, Chan TM, Cheng IK. Clinical and pathological characteristics of patients with glomerular diseases at a university teaching hospital: 5-year prospective review. Hong Kong Med J 1999;5(3):240-4.

[13] Shawarby M, Tamimi DA, Mueilo SA, et al. A clinicopathologic study of glomerular disease: experience of the King Fahd Hospital of the University, Eastern Province, Saudi Arabia. Hong Kong J Nephrol 2010;12(1):20-30.
[14] Chandra H, Chandra S. Patterns of glomerulonephritis in Uttarakhand state-13 years' single centre experience at teaching institute. Indian Medical Gazette 2013:220-3. 